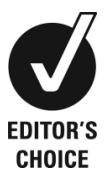

CHOICE

\title{
First use of bedaquiline in a patient with XDR-TB in Singapore
}

\author{
Angeline Poh-Gek Chua, ${ }^{1}$ Grace Si-Ru Hoo, ${ }^{2}$ Cynthia Bin-Eng Chee, ${ }^{3}$ \\ Yee Tang Wang ${ }^{3}$
}

${ }^{1}$ TB Control Unit, Tan Tock Seng Hospital, Singapore, Singapore

${ }^{2}$ Department of Pharmacy, Tan Tock Seng Hospital, Singapore, Singapore ${ }^{3}$ Department of Respiratory Medicine, TB Control Unit, Tan Tock Seng Hospital, Singapore, Singapore

\section{Correspondence to} Dr Angeline Poh-Gek Chua, angeline_pg_chua@ttsh.com. $\mathrm{sg}$

Accepted 28 August 2015

\section{SUMMARY}

Drug-resistant tuberculosis (TB) continues to pose a threat to global control of TB: $3.5 \%$ of new and $20.5 \%$ of previously treated TB cases were estimated to have multidrug-resistant (MDR)-TB in 2013. Approximately $9 \%$ of patients with MDR-TB had extensively drug-resistant (XDR)-TB. A 30-year-old Vietnamese woman previously treated for TB in her home country presented with 5 months of cough and shortness of breath 1 year after migrating to Singapore. Xpert MTB/Rif testing showed rpoB gene mutation. Phenotypic drug susceptibility testing revealed XDR-TB Second and third-line TB drugs were commenced. To strengthen the efficacy of her treatment regimen, the novel anti-TB drug bedaquiline was obtained for the patient on compassionate grounds. We report the first use in Singapore of bedaquiline for the treatment of XDR-TB.

\section{CASE PRESENTATION}

The patient, a 30-year-old Vietnamese woman, moved to Singapore in June 2013. She presented to a general practitioner in May 2014 with productive cough for 5 months and exertional shortness of breath. She had been treated for pulmonary tuberculosis (TB) in Vietnam for 1 year in 2012-2013. There was no history of diabetes or other medical conditions except for a breast augmentation procedure. The patient was a non-smoker and was HIV-negative. A chest X-ray showed extensive opacification of the left lung, right upper zone opacities and air-fluid level in the left hemithorax (figure 1). The patient was promptly referred and admitted to a public hospital for further management.

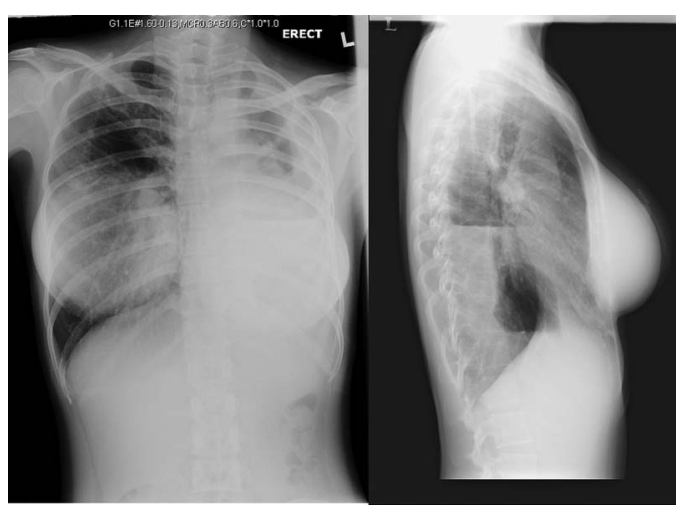

Figure 1 Chest X-ray.
On physical examination, the patient was febrile with a temperature of $37.7^{\circ} \mathrm{C}$. Her vital signs were stable and she weighed $37 \mathrm{~kg}$. There was decreased air entry on auscultation of the left chest.

A sputum AFB smear was positive (3+). HAIN Genotype MTBDRplus 2.0 revealed katG and rpoB mutations indicating resistance to isoniazid and rifampicin.

CT thorax showed extensive scarring, bronchiectasis and loss of lung volume in the left lung with formation of large cavities and pleural thickening. There was air-fluid level suggesting left hydropneumothorax and suspicion of a bronchopleural fistula. Multiple centrilobular nodules were seen in the right lung, with some of them in tree-in-bud pattern, most prominently in the right middle lobe and superior segment of the right lower lobe. There were two cavitary lesions in the right upper lobe, measuring 1.4 and $0.8 \mathrm{~cm}$ in diameter (figure 2).

The patient revealed that she had been treated with cycloserine, prothionamide, PAS and ofloxacin in Vietnam, which she had self-administered from June 2012 until June 2013. In view of this drug history, suspicion of extensively drug-resistant (XDR)-TB was high and treatment with capreomycin $0.5 \mathrm{mg}$ every morning, moxifloxacin $400 \mathrm{mg}$ every morning, linezolid $600 \mathrm{mg}$ every morning, clofazimine $50 \mathrm{mg}$ every morning, ethambutol $700 \mathrm{mg}$ every morning and pyrazinamide $750 \mathrm{mg}$ every morning was prescribed (figure 3 ). To provide the patient with the best available treatment options, an application to obtain bedaquiline (BDQ) via the manufacturer's compassionate use programme was made at the same time as the initiation of therapy.

The second-line HAIN Genotype MTBDRsl test showed rrs and gyr mutations indicating resistance to aminoglycosides and fluoroquinolones (FQLs). Phenotypic drug susceptibility tests (DST) using the non-radiometric Mycobacterial Growth Indicator Tube method subsequently reported resistance to isoniazid, rifampicin, streptomycin, pyrazinamide, kanamycin, ofloxacin, ethionamide, PAS, levofloxacin and amikacin, and sensitivity to ethambutol, clofazimine and capreomycin. The microbroth dilution method using Trek Sensititre plates, revealed sensitivity to cycloserine and resistance to moxifloxacin at $2 \mu \mathrm{g} / \mathrm{mL}$. Based on these results, pyrazinamide was discontinued and cycloserine introduced. Augmentin was included to strengthen the treatment regimen (figure 3 ).

A supply of BDQ for the patient arrived in Singapore 13 weeks after application, that is, 13 weeks after the patient started XDR-TB 


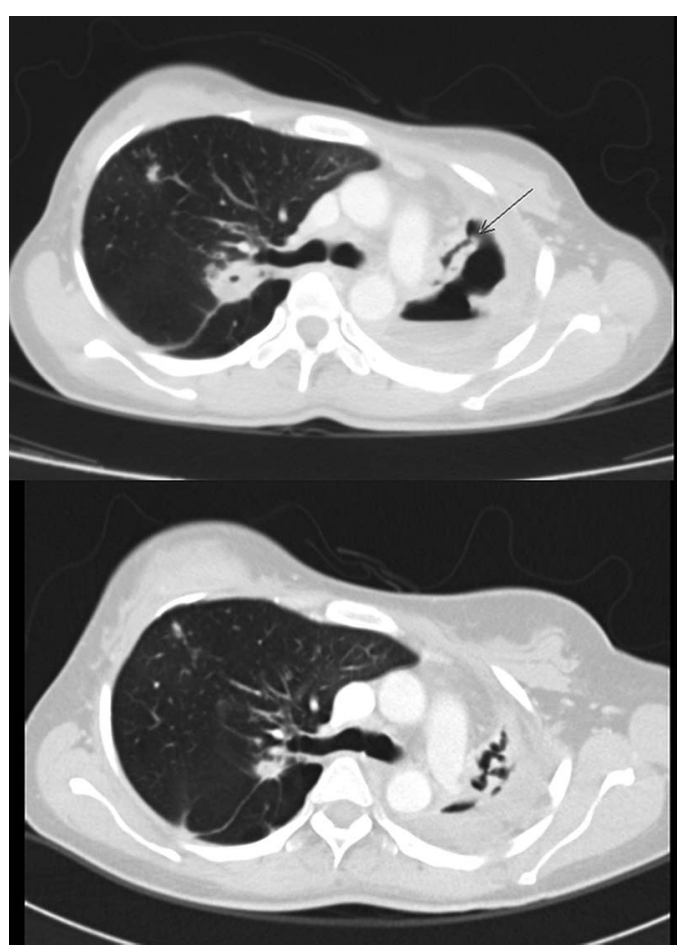

Figure 2 CT thorax on 31 May 2014 before (above) and on 8 October 2014, 16 weeks after initiation of extensively drug-resistant (XDR)-tuberculosis (TB) medication (below). The arrow indicates a bronchopleural fistula.

treatment. She received BDQ $400 \mathrm{mg}$ every morning for 2 weeks, followed by $200 \mathrm{mg}$ three times a week with food for 22 weeks, according to the manufacturer's advice, over a background regimen of capreomycin, ethambutol, linezolid, clofazimine, augmentin and cycloserine. Moxifloxacin was stopped upon commencement of BDQ in accordance with the manufacturer's recommendation in view of the risk of QT prolongation when given in conjunction with BDQ.

The patient tolerated all medications with improvement in appetite and weight. There were no neurological side-effects and she remained in good spirits despite prolonged confinement in an airborne infection isolation room. Baseline ECG was normal with $\mathrm{QT}_{\mathrm{c}} 444 \mathrm{~ms}$. Monthly ECGs did not reveal any QT prolongation. Monthly serum creatinine and electrolytes remained normal. Mild transaminitis remained stable at $<2 \times$ elevated. There was no haematological suppression. However the patient developed asymptomatic hypereosinophilia that was possibly caused by clofazimine which was discontinued at the 29th week of treatment.

Fortnightly sputum sampling for AFB smears and culture showed conversion to sputum smear negativity at 8 weeks and Mycobacterium tuberculosis culture negativity at 14 weeks of treatment. However, development of resistance to capreomycin was noted in her MTC isolate at 5 weeks of treatment. CT thorax at 4 months of treatment showed no change in left lung findings with stable left hydropneumothorax due to a bronchopleural fistula, with scarring and volume loss. The previously noted cavitary lesion in the right upper lobe showed interval decrease in size, with scarring and mild bronchiectatic changes in the apical segment of the right upper lobe.

To accord the patient the best chance of treatment success, and to minimise the risk of relapse, left pneumonectomy was attempted at 7 months of treatment. However, this was not possible as the left pulmonary hilum, left lung and heart were found to be densely adhered and inseparable at the time of surgery.

At the time of writing, the patient has received 10 months of XDR-TB treatment including 24 weeks of BDQ. Her sputum mycobacterial cultures have remained negative since the 14 th week of treatment.

\section{GLOBAL HEALTH PROBLEM LIST}

Early diagnosis of drug-resistant $\mathrm{TB}$ is important to enable appropriate treatment and avoid escalating the degree of resistance. Treatment of drug-resistant TB with currently available second-line TB drugs is long, poorly tolerated and has a poor outcome. New drugs to be administered under supervision are urgently needed.

\section{GLOBAL HEALTH PROBLEM ANALYSIS}

Multidrug-resistant (MDR) and XDR-TB pose serious threats to global TB control. ${ }^{1}$ XDR-TB is defined as resistance to any FQL

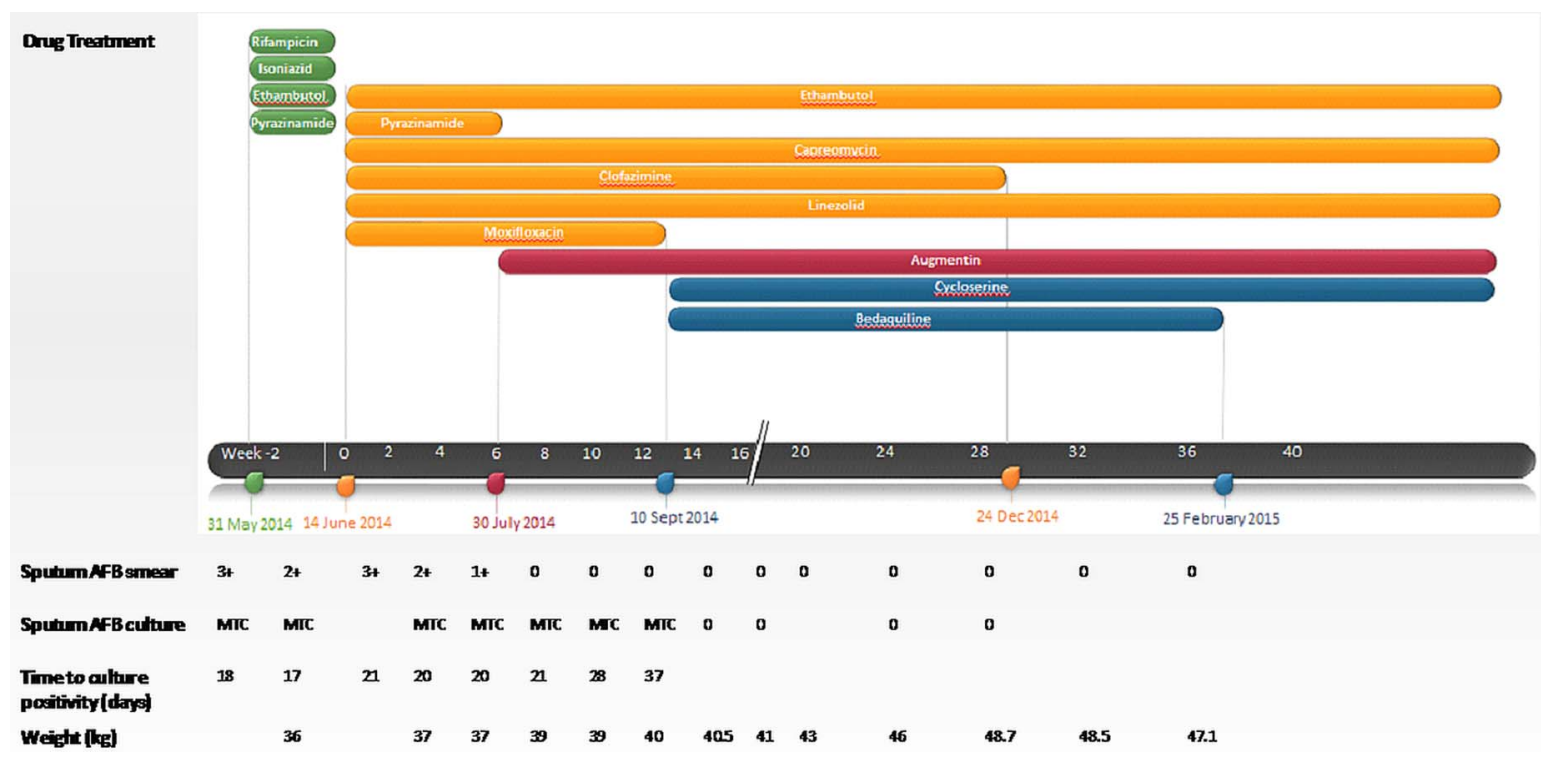

Figure 3 Summary of drug treatment, microbiological responses, time to culture conversion and patient's weight during the course of treatment. 
and to at least one of three second-line injectable drugs (capreomycin, kanamycin, amikacin), in addition to MDR-TB. MDR-TB is resistance to at least isoniazid and rifampicin, the two most important TB drugs. ${ }^{2}$ Drug resistance surveillance data indicate that globally, $3.5 \%$ of new and $20.5 \%$ of previously treated TB cases were estimated to have had MDR-TB in 2013. This translates into an estimated 480000 people having developed MDR-TB in 2013. On average, 9\% of patients with MDR-TB had XDR-TB. ${ }^{3}$

XDR-TB is man-made. Inadequate drug treatment (which may be due to inadequate regimens, inadequate supply/quality of drugs or inadequate drug intake) ${ }^{4}$ selects out drug resistant strains that subsequently proliferate and become MDR strains. Secondary MDR-TB eventually could be disseminated to contact cases who then acquire primary MDR-TB. ${ }^{5}{ }^{6}$ MDR-TB precedes XDR-TB. ${ }^{7}$ Directly observed therapy (DOT) has been shown to reduce the rate of drug resistance in the community. ${ }^{8}$

This patient had a history of self-administered treatment with second-line TB drugs in Vietnam before arriving in Singapore. Hence diagnostic tests for drug-resistance TB using a HAIN Genotype MTBDR assay were done early. Dalton et $\mathrm{al}^{7}$ reported in the Preserving Effective TB Treatment Study (PETTS) that previous treatment with second-line drugs was a strong risk factor for resistance to these drugs including XDR-TB.

Treatment of MDR/XDR-TB is costly, ${ }^{9}$ toxic $^{10}{ }^{11}$ and prolonged. Under WHO guidelines, ${ }^{12}$ the recommended duration of therapy can be up to 2 years. WHO recommended regimens contain pyrazinamide together with a later-generation FQL, ethionamide or prothionamide, cycloserine or para-aminosalicylic acid and a second-line injectable drug. A conventional DST for first and second-line anti-TB drugs, performed in quality assured laboratories, is required to tailor treatment regimes but takes more than 1 month to produce definitive results. In the meantime, the WHO stepwise approach, taking into account the drugs used previously by the patient, the most likely resistance pattern in the setting where the infection most likely occurred, and the resistance pattern of the index case, if known, is recommended for designing an effective MDR-TB regimen.

Treatment of XDR-TB is associated with a higher rate of treatment failure and mortality than MDR-TB. ${ }^{4}$ Our patient showed resistance to all FQLs tested. There is convincing evidence that FQLs are associated with better prognosis when used for MDR-TB. ${ }^{14}{ }^{15}$ Falzon et $a l^{16}$ in analysing the impact of additional resistance to FQLs and/or second-line injectable drugs on treatment outcome, found that among these two drug families which constitute the main treatment for patients with MDR-TB, FQLs, especially the later generation ones, are better than second-line injectables. MDR-TB patients infected with strains without additional resistance had $64 \%$ treatment success compared to $56 \%, 48 \%$ and $40 \%$ in those with MDR-TB with additional resistance to second-line injectables only, additional resistance to FQL alone, and resistant to FQL and second-line injectables (XDR-TB), respectively. They found that in XDR-TB patients, treatment success was highest if at least six drugs were used in the intensive phase and four drugs in the continuation phase. The odds of success in XDR-TB patients was maximised when the intensive phase lasted for 6.6-9 months and the total duration of treatment was 20.1-25 months.

BDQ is the first new class of anti-TB agents to be approved in 40 years. It is a diarylquinoline and exerts bactericidal and sterilising activity against M. tuberculosis. ${ }^{17}$ BDQ targets the central region of the $c$ subunit of the enzyme ATP synthase, interfering with the mycobacterial conversion of ADP into ATP by interrupting transmembrane and central stalk rotation of the proton pump, leading to inadequate synthesis of ATP, resulting in its death. ${ }^{18}$ BDQ's novel mechanism of action suggests that it should have activity against resistant organisms. Because of this mechanism of action, it is bactericidal against even dormant or non-replicating bacilli. ${ }^{19}$ BDQ is $2 \times 10^{4}$ more avid for mycobacterial than for mammalian ATP synthase. ${ }^{20}$

In his French cohort, Guglielmetti et $a l^{21}$ found that at 6 months of BDQ treatment, culture conversion was achieved in $97 \%$ of patients, with a median time to culture conversion of 85 days. Diacon et $a l^{22}$ had also found that BDQ, when given together with a background regimen, led to faster culture conversion and higher rates of culture conversion at 6 months of treatment compared to a background regimen and placebo. This suggests BDQ's potential capacity to significantly reduce the treatment period and the debilitating and dangerous adverse effects associated with some of the existing second-line anti-TB drugs, as well as to improve adherence, achieve higher cure rates, and preserve the activity of the background regimen. ${ }^{23}$

Our results agreed that BDQ-containing regimens contributed to the reduced time to culture conversion and radiological improvements. BDQ was well-tolerated in our patient with minimal side effects. Although we were concerned that our patient, who was taking concomitant clofazimine and BDQ, would experience side-effects (particularly QT prolongation), no additional adverse events or QT prolongation were noted. Other common side-effects of hyperuricaemia, elevated aminotransferases, nausea, arthralgia or headache previously reported with BDQ, were not noted here. ${ }^{24}$ BDQ's lack of toxicity in this case meant that no treatment interruption was required. We applied to Janssen Therapeutics (the manufacturers of BDQ), via our hospital pharmacy, to enrol our patient in their 'compassionate use' programme. After a detailed and lengthy process, rightly so to prevent misuse of the drug, approval was obtained. $^{25} 26$ We next had to get clearance from the Health Sciences Authority, the regulatory authority in Singapore.

The current recommended duration of BDQ is 24 weeks due to the lack of trial data supporting its continuation after this period. It should be noted that BDQ has a very large apparent volume of distribution and has a markedly prolonged terminal half-life (5.5 months), which reflects the slow release of the compound from peripheral tissue compartments. ${ }^{27}$ Therefore, there is lingering activity of the drug in the body despite discontinuation. BDQ's long half-life is a double-edged sword. Excessive half-life will increase the risks to the patient when BDQ does cause toxicity and will increase the risks of the emergence of BDQ resistance if drugs are discontinued or have mismatched half-lives and long dosing intervals. ${ }^{27} 28$

Of note, BDQ was approved based on trials that evaluated the surrogate marker of culture conversion rather than the gold standard of durable cure after completion of therapy. ${ }^{29}$ Potential QT prolongation also limits its use in patients with cardiac conduction abnormalities and in patients receiving other QT-prolonging agents, such as macrolides, FQLs and azole antifungals. Because BDQ is metabolised by CYP3A4, its blood concentration and therapeutic effect may be altered by co-administration with CYP3A4 inducers or inhibitors. ${ }^{30}$ Drug interactions warrant close monitoring, and will limit utility of BDQ particularly in patients receiving rifamycins and/or antiretroviral therapy. Finally, the cost of a 24-week course of therapy with BDQ is US\$36000 (average wholesale price), which is higher than the cost of other second-line agents commonly used in the treatment of MDR-TB.

Recently, due to renewed interest in the anti-leprosy drug clofazimine which is being re-purposed for the treatment of 
MDR-TB, cross-resistance mechanisms have been discovered between clofazimine and BDQ. ${ }^{31-33}$ The arrival of new drugs will enhance our capacity to cure $\mathrm{TB}$, but clinicians must be aware of the ability of $M$. tuberculosis to generate resistance. ${ }^{34}$ Against this threat of emerging drug resistance, we must balance the need to get better drugs for our patients and their rational use within properly designed background regimens and public health policies.

\section{Learning points}

- Suspect drug resistant tuberculosis (TB) if there is a history of previously treated TB especially if TB treatment was not administered under directly observed therapy.

- Use rapid molecular diagnostic tests like the Cepheid Xpert MTB/RIF and HAIN Genotype MTBDRplus when drug-resistant TB is suspected, and send specimens for mycobacterial culture and phenotypic drug susceptibility testing so an appropriate multidrug-resistant (MDR)/ extensively drug-resistant (XDR)-TB drug regimen can be instituted.

- Bedaquiline, a new agent with bactericidal and sterilising activity against Mycobacterium tuberculosis, is effective against MDR/XDR-TB when given together with a background regimen, and is well tolerated and safe if there is awareness of drug interactions and precautions are taken to avoid potential QT prolongation.

- Be aware of new mechanisms of cross-resistance between TB drugs.

- National TB programmes need new drugs that can be used within short, effective and safe regimens.

Acknowledgements We would like to thank the nurses of the Communicable Diseases Centre (wards 72 and 75 ) for taking care of the patient. We thank Janssen, a division of Johnson \& Johnson Pte Ltd, for supplying the novel drug bedaquiline under their compassionate use programme We acknowledge the staff of the Central TB Laboratory for carrying out the sputum tests including drug sensitivity testing.

Contributors AP-GC, GS-RH and CB-EC contributed equally to the content. CB-EC and YTW checked and reviewed manuscript before submission.

Competing interests None declared.

Patient consent Obtained.

Provenance and peer review Not commissioned; externally peer reviewed.

\section{REFERENCES}

1 Gandhi NR, Nunn P, Dheda K, et al. Multidrug-resistant and extensively drug-resistant tuberculosis: a threat to global control of tuberculosis. Lancet 2010;375:1830-43.

2 World Health Organization. Definitions and reporting framework for tuberculosis2013 revision. World Health Organization, 2013. WHO/HTM/TB/2013.2.

3 World Health Organisation. Drug-resistant TB. Surveillance and response. Supplement: Global Tuberculosis Report 2014. World Health Organisation, 2014. WHO/HQ/TB/2014.12.

4 Lange C, Abubakar I, Alffenaar J, et al. Management of patients with multidrug-resistant/extensively drug-resistant tuberculosis in Europe: a TBNET consensus statement. Eur Respir J 2014;44:23-63.

5 Zumla A, Raviglione M, Hafner R, et al. Tuberculosis. N Engl J Med 2013;368:745-55.

6 Caminero JA, Sotgiu G, Zumla A, et al. Best drug treatment for multidrug-resistant and extensively drug-resistant tuberculosis. Lancet Infect Dis 2010;10:621-9.
7 Dalton T, Cegielski P, Akksilp S, et al. Prevalence of and risk factors for resistance to second-line drugs in people with multidrug-resistant tuberculosis in eight countries: a prospective cohort study. Lancet 2012;380:1406-17.

8 Weis SE, Slocum PC, Blais FX, et al. The effect of directly observed therapy on rates of drug resistance and relapse in tuberculosis. N Engl J Med 1994;330:179-84.

9 Phua CK, Chee CBE, Chua APG, et al. Managing a case of extensively drug-resistant (XDR) pulmonary tuberculosis in Singapore. Ann Acad Med Singapore 2011:40:132-5.

10 Nathanson E, Gupta R, Huamani P, et al. Adverse events in the treatment of multidrug-resistant tuberculosis: results from the DOTS-Plus initiative. Int J Tuberc Lung Dis 2004;8:1382-4.

11 Van der Walt M, Lancaster J, Odendaal R, et al. Serious treatment related adverse drug reaction among anti-retroviral naive MDR-TB patients. PLOS ONE 2013;8: e58817.

12 Falzon $\mathrm{D}$, Jaramillo $\mathrm{E}$, Schünemann $\mathrm{HJ}$, et al. WHO guidelines for the programmatic management of drug-resistant tuberculosis: 2011 update. Eur Respir $J$ 2011;38:516-28.

13 Migliori GB, Sotgiu G, Gandhi NR, et al. Drug resistance beyond extensively drug-resistant tuberculosis: individual patient data meta-analysis. Eur Respir J 2013;42:169-79.

14 Johnston JC, Shahidi NC, Sadatsafavi M, et al. Treatment outcome of multi-drug resistant tuberculosis: a systematic review and meta-analysis. PLOS ONE 2009;4: e6914.

15 Chiang CY, Enarson DA, Yu MC, et al. Outcome of pulmonary multidrug-resistant tuberculosis: a 6-year follow-up study. Eur Respir J 2006;28:980-5.

16 Falzon D, Gandhi N, Migliori GB, et al. Resistance of fluoroquinolones and second-line injectable drugs: impact on multi-drug resistant TB outcomes. Eur Respir J 2013;42:156-68.

17 Singh H, Natt NK, Garewal N, et al. Bedaquiline: a new weapon against MDR and XDR-TB. Int J Basic Clin Pharmacol 2013;2:96-102.

18 Andries $\mathrm{K}$, Verhasselt P, Guillemont J, et al. A diarylquinoline drug active on the ATP synthase of Mycobacterium tuberculosis. Science 2005;307:223-7.

19 Koul A, Vranckx L, Dendouga N, et al. Diarylquinolines are bactericidal for dormant mycobacteria as a result of disturbed ATP homeostasis. J Biol Chem 2008;283:25273-80.

20 Haagsma AC, Abdillahi-Ibrahim R, Wagner MJ, et al. Selectivity of TMC207 towards mycobacterial ATP synthase compared with that towards the eukaryotic homologue. Antimicrob Agents Chemother 2009;53:1290-2.

21 Guglielmetti L, Le Du D, Jachym M, et al. Compassionate use of Bedaquiline for the treatment of multidrug-resistant and extensively drug-resistant tuberculosis: interim analysis of a French cohort. Clin Infect Dis 2015;60:188-94.

22 Diacon AH, Pym A, Grobusch MP et al. Multidrug-resistant tuberculosis and culture conversion with Bedaquiline. N Engl J Med 2014;371:723-32.

23 Cohen J. Approval of novel TB drug celebrated-with restraint. Science 2013;339:130.

24 World Health Organization. The use of bedaquiline in the treatment of multidrug-resistant tuberculosis: interim policy guidance. Document WHO/HTM/TB/ 2013.6. Geneva, World Health Organization, 2013.

25 Compassionate Use Programme of TMC207 in patients with Extensively Drug Resistant(XDR) or Pre-XDR Mycobacterium tuberculosis (MTB) Pulmonary Infection. Guidelines for Physicians and Pharmacists. To access, contact RA-CNTNL-JANSSENCU@ITS.JNJ.COM.

26 Challenges for Compassionate Use of New TB Drugs. http://www.resisttb.org/? page_id=1371 (accessed 6 Jul 2013).

27 Diacon AH, Donald PR, Pym A, et al. Randomized pilot trial of eight weeks of bedaquiline (TMC207) treatment for multidrug-resistant tuberculosis: long-term outcome, tolerability, and effect on emergence of drug resistance. Antimicrob Agents Chemother 2012;56:3271-6.

28 Diacon AH, Pym A, Grobusch M, et al. The diarylquinoline TMC207 for multidrug-resistant tuberculosis. N Engl J Med 2009;360:2397-405.

29 Avorn J. Approval of a tuberculosis drug based on a paradoxical surrogate measure. JAMA 2013;309:1349-50.

30 Sirturo (bedaquiline) [product information]. Titusville, NJ: Janssen Therapeutics, 2012.

31 Hartkoorn RC, Uplekar S, Cole ST. Cross resistance between clofazimine and bedaquiline through upregulation of MmpL5 in Mycobacterium tuberculosis. Antimicrob Agents Chemother 2014;58:2979-81.

32 Andries K, Villellas C, Coeck N, et al. Acquired resistance of Mycobacterium tuberculosis to bedaquiline. PLOS ONE 2014;9:e102135.

33 Tiberi $S$, De Lorenzo $S$, Centis $R$, et al. Bedaquiline in MDR/XDR-TB cases: first experience on compassionate use. Eur Respir J 2014;43:289-92.

34 Somoskovi A, Bruderer V, Hömke R, et al. A mutation associated with clofazimine and bedaquiline cross-resistance in MDR-TB following bedaquiline treatment. Eur Respir J 2015;45:554-5. 
Copyright 2015 BMJ Publishing Group. All rights reserved. For permission to reuse any of this content visit http://group.bmj.com/group/rights-licensing/permissions.

BMJ Case Report Fellows may re-use this article for personal use and teaching without any further permission.

Become a Fellow of BMJ Case Reports today and you can:

- Submit as many cases as you like

- Enjoy fast sympathetic peer review and rapid publication of accepted articles

- Access all the published articles

- Re-use any of the published material for personal use and teaching without further permission

For information on Institutional Fellowships contact consortiasales@bmjgroup.com

Visit casereports.bmj.com for more articles like this and to become a Fellow 